\title{
Insightful crystal-structure classification using deep learning
}

\author{
Angelo Ziletti $^{1}{ }$, Matthias Scheffler ${ }^{1}$, And Luca M. Ghiringhelli ${ }^{1}$ \\ ${ }^{1}$ Fritz Haber Institute of the Max Planck Society, Berlin, Germany \\ ziletti@fhi-berlin.mpg.de
}

\begin{abstract}
Big data is emerging as a new paradigm in materials science. A vast amount of three-dimensional structural data is provided by both computational repositories (e.g. http://nomad-coe.eu) and experiments (e.g. atom probe tomography). Computational methods that automatically and efficiently detect longrange order are of paramount importance for materials characterization and analytics. Current methods are either not stable with respect to defects, or base their representation on local atomic neighbourhoods, which in turn makes it difficult to detect "average" longe-range order. In the proposed approach, for a given crystal structure we first calculate its diffraction pattern, expand it on spherical harmonics, and then use a neural-network model to obtain a compact, low-dimensional representation. We apply this workflow to a subset of materials from the Novel Materials Discovery (NOMAD) Archive, and show that our deep-learning-based approach compactly encodes structural information, is robust to defects (e.g. point defects, and/or strain), and allows to build easily interpretable structural-similarity maps. This work received funding from the NOMAD Laboratory, a European Center of Excellence.
\end{abstract}

